\title{
Evaluación ultrasonográfica de la tiroides en crías de vacas lecheras tratadas con yodo en el preparto
}

\author{
Ultrasonographic evaluation of the thyroid gland in offspring of dairy cows \\ supplemented with iodine during the prepartum
}

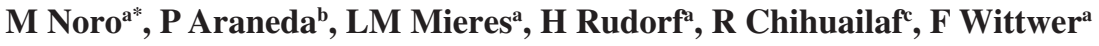 \\ anstituto Ciencias Clínicas Veterinarias, Universidad Austral de Chile, Valdivia, Chile.

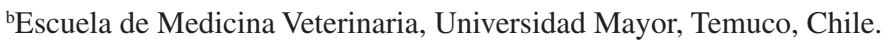 \\ 'Escuela de Medicina Veterinaria, Universidad Católica de Temuco, Temuco, Chile.
}

\begin{abstract}
SUMMARY
The aim of this work was to determine the size of the thyroid gland and the weight gain of calves born from dairy cows supplemented with iodine (I) during prepartum. Fourteen Holstein-Friesian calves were used. They were born from 7 cows supplemented with a single dose of I (I-S, $1.6 \mathrm{~g} / \mathrm{cow}$, Lipiodol ${ }^{\circledR}$ ) at seven month of pregnancy and 7 non supplemented cows (I-C). Cows grazed a low I content pasture (0.19ppm) until calving. The thyroid gland of the calves was examined by ultrasonography at days 36 and $128( \pm 7.5)$ of age. Calves were weighted at 26 and $110( \pm 7.5)$ days of age and the daily weight gain (DWG) was determined. The thickness of the isthmus of the thyroid at 36 days of age were similar between groups (P $>0.05$ ). A reduction in thickness was observed in both groups at 128 days, more pronounced in I-C group $(\mathrm{P}<0.05)$. The left lobule was larger at 36 days of age in the I-C group than in I-S group $(\mathrm{P}<0.05)$. An increase in size of the left lobule was found in both groups after 128 days $(\mathrm{P}<0.05)$. The size of the right lobule was similar for both groups $(\mathrm{P}>0.05)$ in both periods. Calves of both groups had a similar weight and DWG at 36 and 128 days of age. It was concluded that a single supplementation with I for pregnant dairy cows grazing a low iodine content pasture induced a reduction in the growth of the left lobule of the thyroid in their offspring without affecting the growth of the calves.
\end{abstract}

Key words: iodine supplementation, calves, thyroid, ultrasonography.

\section{RESUMEN}

El objetivo del estudio fue determinar ultrasonográficamente el tamaño de la glándula tiroides y la ganancia de peso de terneros crías de vacas suplementadas con yodo (I) en el preparto. Se utilizaron 14 terneros Holstein-Friesian, crías de 7 vacas suplementadas con I a los 7 meses de gestación (I-S, 1,6 g/vaca, Lipiodol ${ }^{\circledR}$ ) y de 7 vacas no suplementadas (I-C), pastoreando praderas con bajo contenido de I. A todos los terneros se les realizó un examen ultrasonográfico de la glándula tiroides a los 36 y $128( \pm 7,5)$ días de edad y se determinó el peso y la ganancia diaria de peso (GPD) desde el nacimiento hasta los 26 y $110( \pm 7,5)$ días de edad. El grosor del istmo de la tiroides a los 36 días de edad fue similar entre grupos $(\mathrm{P}>0,05)$, disminuyendo a los 128 días de edad de forma más marcada en el grupo I-C $(\mathrm{P}<0,05)$. El tamaño del lóbulo izquierdo fue mayor en los terneros I-C que en los I-S a los 36 días de edad ( $\mathrm{P}<0,05)$, aumentando en ambos a los 128 días $(\mathrm{P}<0,05)$. El tamaño del lóbulo derecho fue similar para ambos grupos, y no se relacionó con el peso vivo $(\mathrm{P}>0,05)$. Los pesos y las GPD de ambos grupos fueron similares. Se concluye que una suplementación con I durante el preparto a vacas mantenidas a pastoreo sobre praderas con bajo contenido de I condicionó en sus crías un menor tamaño del lóbulo izquierdo de la glándula tiroides, sin alterar su peso o la GPD.

Palabras clave: tiroides, ultrasonido, terneros, yodo.

\section{INTRODUCCIÓN}

La glándula tiroides del bovino está localizada caudal a la laringe, siendo conformada por un lóbulo derecho, uno izquierdo y un istmo que los conecta (Seirferle 1975). Como glándula endocrina secreta las hormonas tiroxina $\left(\mathrm{T}_{4}\right)$, triiodotironina $\left(\mathrm{T}_{3}\right)$ y calcitonina. $\mathrm{La} \mathrm{T}_{3}$ proviene de una deionización de la $\mathrm{T}_{4}$, actúa regulando

Aceptado: 20.12.2012.

*mirelanoro@gmail.com el metabolismo intermediario de los carbohidratos, proteínas y lípidos; la temperatura, el crecimiento y desarrollo corporal, las funciones reproductiva y musculares, además, de controlar la tasa de oxidación celular (Underwood y Suttle 1999). La calcitonina por su parte es una hormona hipocalcemiante. El yodo (I) es parte de la estructura de la $\mathrm{T}_{4} \mathrm{y} \mathrm{T}_{3}$, por lo que su carencia nutricional inhibe la síntesis de estas hormonas (Underwood y Suttle 1999, Eiler 2006). Al respecto se describe que el requerimiento diario de I en las vacas lecheras es de $0,33 \mathrm{ppm} /$ $\mathrm{kg}$ materia seca (NRC 2001). La inducción experimental de una carencia de I produce hiperplasia de la glándula 
tiroides o bocio en animales jóvenes, siendo éste el signo clínico más frecuentemente observado (McCoy y col 1997). Una glándula tiroides activa, presenta reservorios coloidales pequeños con células foliculares alargadas, a diferencia de una glándula inactiva, como la observada en el bocio, en que las células son aplanadas y los folículos se encuentran distendidos con el coloide (Eiler 2006).

Durante el inicio de la gestación la carencia de $\mathrm{T}_{3} \mathrm{y}$ $\mathrm{T}_{4}$ causada por el hipotiroidismo ejerce un efecto negativo sobre la diferenciación y el crecimiento del esqueleto, inhibiendo el desarrollo fetal; efectos que persisten posterior al nacimiento y que conducen a un retardo en el crecimiento (Eiler 2006).

Los forrajes del sur de Chile presentan bajas concentraciones de I (Contreras y col 2003), situación que se asocia con la presentación de hipotiroidismo subclínico; condición detectada por las bajas concentraciones sanguíneas de $\mathrm{T}_{3}$ y $\mathrm{T}_{4}$ en rebaños lecheros (Contreras y col 2005). Si bien la concentración sérica de $\mathrm{T}_{4}$ reflejaría el estatus de I y la funcionalidad de la tiroides en los animales domésticos, esta relación está más descrita en ovejas que en otras especies. Por otra parte, su utilidad en el diagnóstico de hipotiroidismo en rumiantes es menos satisfactoria que en humanos ya que diversos factores como la edad, estado reproductivo, lactancia, período del año afectan su concentración (Underwood y Suttle 1999). Es así como en terneros los valores séricos de $\mathrm{T}_{3} \mathrm{y} \mathrm{T}_{4}$ disminuyen con la edad con valores para terneros de 1 día de edad de 1,2 - 1,7 y $35-55 \mathrm{ng} / \mathrm{mL}$ para $\mathrm{T}_{3}$ y $_{4}$ respectivamente y de $0,6-1,0$ y $12-25 \mathrm{ng} / \mathrm{mL}$ en terneros de 6 semanas (Puls 1994). De igual forma se describe que los terneros recién nacidos tienen una concentración tres veces mayor a la de sus madres, por lo que una relación vaca:cría $<2,5$ sería sugerente de hipotiroidismo en el ternero (MCoy y col 1997).

La carencia de I en vacas puede afectar el desarrollo de sus crías aumentando el tamaño de la tiroides en respuesta compensatoria para compensar la producción disminuida de $\mathrm{T}_{3}$ y $\mathrm{T}_{4}$ (Underwood y Suttle 1999). La evaluación de la morfología y el tamaño de las estructuras de la glándula tiroides mediante ultrasonografía se ha utilizado para monitorear el tratamiento del hipotiroidismo en perros (Taeymans y col 2007), así como del hipertiroidismo en gatos (Barberet y col 2010, Wiesner y col 1991, Bromel y col 2006). De este modo, esta técnica podría ser útil para evaluar la glándula tiroides en rumiantes y su respuesta al tratamiento con I.

El objetivo de este estudio fue evaluar el tamaño de la glándula tiroides y la ganancia de peso de terneros crías de vacas suplementadas con I en el preparto.

\section{MATERIAL Y MÉTODOS}

\section{ANIMALES}

Se utilizaron 14 vacas Holstein-Friesian con 7 meses de preñez, pertenecientes al predio lechero de INIA Cari- llanca, Temuco, Región de la Araucanía, Chile. El rebaño preparto se mantuvo en pastoreo de praderas mixtas fertilizadas con predominio de Lolium sp., trébol (Trifolium $s p$.) y alfalfa (Medicago sp.), con bajo contenido de I $(0,19$ $\mathrm{mg} / \mathrm{kg} \mathrm{MS}$ ). Además en las últimas tres semanas pre parto fueron suplementadas con ensilaje de pradera, maíz (Zea mays) y concentrado energético comercial (Cisterna $\left.{ }^{\circledR}\right)$.

\section{DISEÑO EXPERIMENTAL}

Las vacas se asignaron aleatoriamente según fecha probable de parto a dos tratamientos: controles (I-C, $\mathrm{n}=$ 7) y suplementadas (I-S, $\mathrm{n}=7)$ con I (1,6 g por animal) empleando Lipiodol ${ }^{\circledR}$ s.c. en dosis única, acorde a las indicaciones del fabricante, suministrada dos meses previo a la fecha estimada de parto.

Los 14 terneros nacidos, 7 machos y 7 hembras se transfirieron a jaulas individuales durante 15 días donde recibieron calostro conservado (4 L/día) durante 10 días. Luego, fueron llevados a jaulas colectivas de $5 \times 5 \mathrm{~m}$, con 7 a 8 terneros por jaula, separados por sexo, donde recibieron $5 \mathrm{~L}$ /día de sustituto lácteo Kalmilac ${ }^{\circledR}$, suministrados equitativamente 2 veces al día (8:30 y 16:00 horas). Además contaban con heno de alfalfa y concentrado de iniciación $\left(\right.$ Cisterna $\left.^{\circledR}\right)$ ad libitum. Luego de alcanzar los 80 $\mathrm{kg}$ de peso vivo fueron mantenidos en jaulas colectivas recibiendo concentrado de crecimiento (Cisterna ${ }^{\circledR}$ ) y alfalfa hasta finalizar el ensayo a los $128 \pm 7,5$ días de edad, productos cuya concentración de yodo no estaba informada.

\section{MUESTREOS}

Los terneros fueron pesados al nacimiento, a los $26 \pm 7,5$ y $110 \pm 7,5$ días de edad en una balanza para determinar la ganancia de peso diaria (GPD) desde el nacimiento hasta los 26 días de edad y desde el nacimiento hasta los 110 días de edad. A los $36 \pm 7,5$ días y posteriormente a los $128 \pm 7$, se realizó un examen ultrasonográfico de la glándula tiroides para establecer el tamaño de los lóbulos derecho e izquierdo y el grosor del istmo empleando un equipo de ultrasonido Scanner 480 de Pie $\mathrm{Medical}^{\circledR}$, con transductor lineal de 7,5 MHz con stand off. El área de cada lóbulo fue calculado en $\mathrm{cm}^{2}$, empleando la forma de elipse en consideración a la forma casi triangular observada a la imagen ecográfica. El grosor del istmo fue medido entre la piel y la tráquea y expresado en $\mathrm{cm}$ (figura 1). Para establecer la precisión de la técnica se determinó el coeficiente de variación $(\mathrm{CV})$ de la misma para las tres estructuras anatómicas mediante mediciones en triplicado.

\section{ANÁLISIS ESTADÍSTICO}

Se determinó la media y desviación estándar (DE) de los datos. La diferencia entre los grupos se determinó mediante la prueba " $\mathrm{t}$ " de Student. La diferencia entre 
ecografías para el tamaño de los lóbulos y el grosor del istmo se determinó mediante una prueba " $\mathrm{t}$ " de Student para datos pareados. Se relacionó el tamaño de las estructuras con el peso de los animales mediante análisis de correlación de Pearson usando el programa estadístico Statistix 8.0 (NH Analytical Software, Roseville, MN; USA) (Statistix 2003), con un nivel de significancia de $95 \%$.

\section{RESULTADOS Y DISCUSIÓN}

\section{VISUALIZACIÓN DE LA GLÁNDULA TIROIDES}

La ultrasonografía permitió identificar las tres estructuras que constituyen la tiroides, siendo una técnica simple y fácil de realizar en terneros bajo condiciones de campo. En todos los terneros fue posible distinguir y medir el istmo y los lóbulos derecho e izquierdo, siendo el lóbulo derecho de menor tamaño que el izquierdo, resultado que diverge con el reportado en otro estudio (Seirferle 1975). En la vista transversal, los lóbulos izquierdo y derecho fueron casi triangulares y conectados por el istmo. Las tres estructuras presentaron similar ecotextura; su ecogenicidad fue levemente menor que el tejido conectivo adyacente y mayor a la de los músculos (figura 1). La determinación en triplicado del tamaño de los lóbulos y grosor del istmo permitió establecer una buena precisión de la técnica, con un CV de 8,0\% para el istmo y de $5,8 \%$ y $6,5 \%$ para los lóbulos izquierdos y derechos, respectivamente.

\section{RESPUESTA A LA SUPLEMENTACIÓN}

El grosor del istmo a los 36 días de edad fue similar entre los grupos I-S y I-C; disminuyendo a los 128 días de edad en ambos grupos ( $r=-0,70$; figura 2$)$, si bien este cambio fue más marcado en I-C ( $\mathrm{P}<0,05$; cuadro 1$)$.

El lóbulo izquierdo fue de menor tamaño en I-S que en I-C a los 36 días de edad ( $\mathrm{P}<0,05$; cuadro 1$) \mathrm{y}$, al contrario de lo observado en el istmo, aumentó su tamaño $(\mathrm{P}<0,05$, cuadro 1) en ambos grupos con el desarrollo de los terneros ( $r=0,51$; figura 2 ), a su vez no se asoció con la GPD. Por otro lado, el tamaño del lóbulo derecho
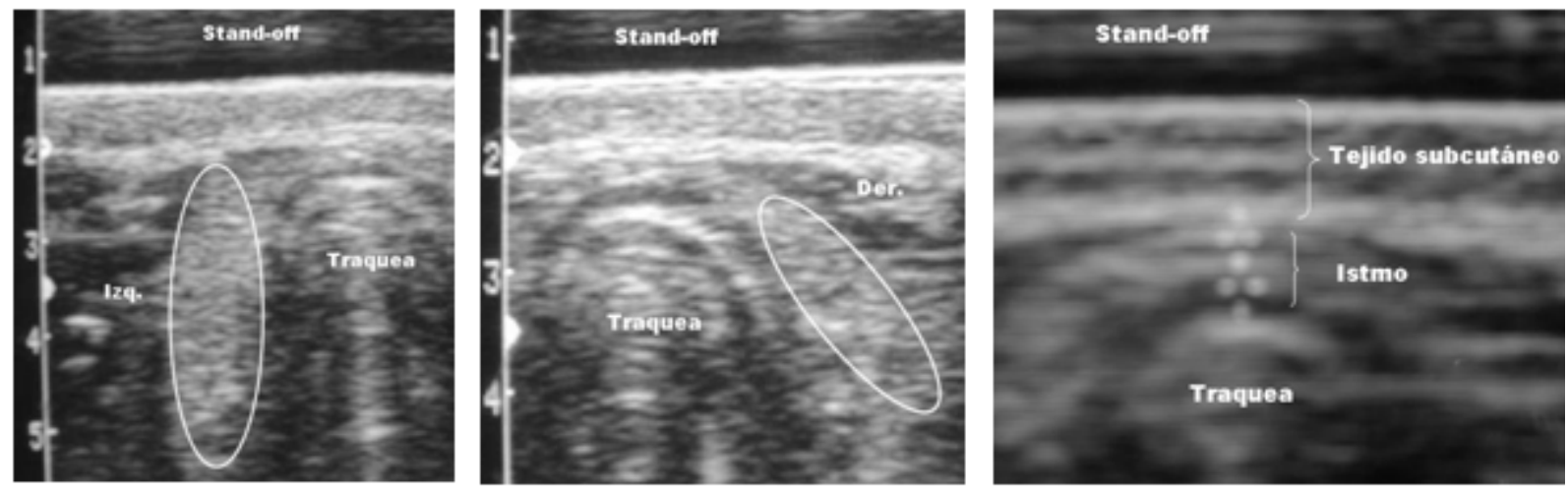

Figura 1. Visión ecográfica de los lóbulos izquierdo (izquierda), derecho (centro) e istmo (derecha) de la glándula tiroides de un ternero de 128 días de edad del grupo control.

Ultrasonography view of the left lobe (left), right (center) and isthmus (right) of the thyroid gland in a calf of 128 days of age.
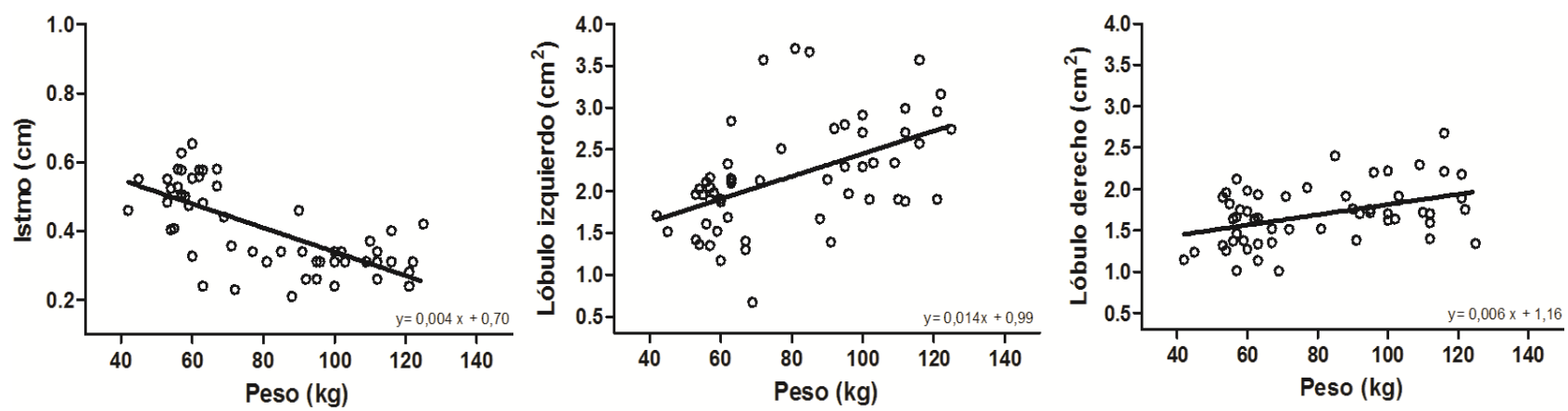

Figura 2. Regresión lineal entre el peso de terneros con el grosor del istmo (izquierda) y tamaño de los lóbulos izquierdo (centro) y derecho (derecha) de la glándula tiroides. I-S: (o) ---; I-C: $(\Delta)-$.

Linear regression between the weight of calves with the thickness of the isthmus (left) and size of the left (center) and right lobes (right) of the thyroid gland. I-S: (o) ----; I-C: $(\Delta)-$. 
Cuadro 1. Valores medios ( \pm DE) del grosor del istmo y área de los lóbulos izquierdo y derecho de la glándula tiroides en crías de vacas lecheras suplementadas con yodo (I-S) durante el preparto y controles (I-C).

Thickness $(\mathrm{X} \pm \mathrm{SD})$ of the isthmus and surface of the left and right lobes of the thyroid gland of calves born from dairy cows supplemented with iodine (I-S) during prepartum and controls (I-C).

\begin{tabular}{lcccc}
\hline & \multicolumn{2}{c}{36 días de edad } & \multicolumn{2}{c}{128 días de edad } \\
\cline { 2 - 5 } & I-C & I-S & I-C & I-S \\
\hline Grosor istmo $(\mathrm{cm})$ & $0,55 \pm 0,10^{\mathrm{a}}$ & $0,50 \pm 0,08^{\mathrm{a}}$ & $0,27 \pm 0,03^{\mathrm{b}}$ & $0,32 \pm 0,06^{\mathrm{c}}$ \\
Área lóbulo izquierdo $\left(\mathrm{cm}^{2}\right)$ & $2,07 \pm 0,40^{\mathrm{a}}$ & $1,59 \pm 0,28^{\mathrm{b}}$ & $2,81 \pm 0,19^{\mathrm{c}}$ & $2,32 \pm 0,44^{\mathrm{c}}$ \\
Área lóbulo derecho $\left(\mathrm{cm}^{2}\right)$ & $1,60 \pm 0,40^{\mathrm{a}}$ & $1,45 \pm 0,28^{\mathrm{a}}$ & $1,85 \pm 0,23^{\mathrm{ab}}$ & $1,86 \pm 0,41^{\mathrm{b}}$ \\
\hline
\end{tabular}

Letras distintas señalan $\mathrm{P}<0,05$.

Cuadro 2. Peso vivo (media \pm DE) a los 26 y 110 días de edad y ganancia de peso diaria (GDP) de las crías de vacas lecheras suplementadas con yodo (I-S) en el preparto y controles (I-C).

Body weight (mean \pm SD) at 26 and 110 days of age and average daily gain (ADG) of calves born from dairy cows supplemented with iodine (S-I) in the prepartum and controls (I-C).

\begin{tabular}{lcccc}
\hline & \multicolumn{2}{c}{26 días } & I-C & I-S \\
\cline { 2 - 5 } & I-C & I-S & $107 \pm 13^{\text {a }}$ & $108 \pm 11^{\text {a }}$ \\
\hline Peso vivo $(\mathrm{kg})$ & $61 \pm 5^{\text {a }}$ & $58 \pm 7^{\text {a }}$ & $603 \pm 90^{\text {a }}$ & $621 \pm 71^{\text {a }}$ \\
\hline GPD desde nacimiento (g/día) & $475 \pm 102^{\text {a }}$ & $394 \pm 106^{\mathrm{a}}$ & 69 \\
\hline
\end{tabular}

Letras distintas señalan $\mathrm{P}<0,05$.

a los 36 días de edad fue similar para ambos aumentando posteriormente sólo en el grupo I-S ( $\mathrm{P}<0,05$; cuadro 1$)$, presentando una baja relación con el peso vivo $(\mathrm{r}=0,45$; $\mathrm{P}<0,05$; figura 2) y sin presentar relación con la GPD.

El menor tamaño del lóbulo izquierdo en los terneros del grupo I-S a los 36 días de edad, coincide con lo observado en niños adolescentes, en quienes el volumen de la tiroides disminuyó con el tratamiento con I (Nakatake y col 2011), efecto posiblemente relacionado a una mayor actividad de la glándula tiroides. Por otro lado, el aumento del tamaño de la tiroides con la edad, especialmente del lóbulo izquierdo, concuerda con lo relatado en niños de 6 a 14 años donde se observó un incremento de la glándula con la edad, efecto que se relacionó con la superficie corporal (Duarte y col 2004), peso, altura e índice de masa corporal (Alves y col 2010).

Los pesos y las GPD de ambos grupos de terneros fueron similares ( $P>0,05$; cuadro 2 ), indicando que la suplementación de las madres con I durante el preparto no influyó sobre el peso vivo y GPD de sus crías hasta los 110 días de edad. Tanto el peso como la GPD de los terneros de ambos los grupos estuvieron dentro de los valores esperados para esa categoría animal (Khan y col 2007).

Se concluye que la suplementación con I durante el preparto a vacas mantenidas a pastoreo sobre praderas con bajo contenido de I condicionó en sus crías un menor tamaño del lóbulo izquierdo de la glándula tiroides, sin alterar su peso o la ganancia posterior de peso.

\section{REFERENCIAS}

Alves MLD, GC Duarte, AM Navarro, EK Tomimori. 2010. Avaliação ultrassonográfica da tireoide, determinação da iodúria e concentração de iodo em sal de cozinha utilizado por escolares de Ribeirão Preto, São Paulo, Brasil. Arq Bras Endocrin Metabol 54, 813-818.

Barberet V, Y Baeumlin, O Taeymans, L Duchateau, K Peremans, I van Hoek, S Daminet, JH Saunders. 2010. Preand posttreatment ultrasonography of the thyroid gland in hyperthyroid cats. Vet Radiol Ultrasound 51, 324-330.

Bromel C, RE Pollard, PH Kass, VF Samii, AP Davidson, RW Nelson. 2006. Comparison of ultrasonographic characteristics of the thyroid gland in healthy small-, medium-, and large-breed dogs. Am J Vet Res 67, 70-77.

Contreras PA, A Ceballos, R Matamoros, F Wittwer. 2003. Contenido de yodo en forrajes de predios lecheros de las Regiones IX y X de Chile. Arch Med Vet 35, 75-79.

Contreras PA, F Wittwer, R Matamoros, IM Mayorga, G van Schaik. 2005. Effect of grazing pasture with a low selenium content on the concentrations of triiodothyronine and thyroxine in serum, and GSH-Px activity in erythrocytes in cows in Chile. $N Z$ Vet $J 53,77-80$.

Duarte GC, EK Tomimori, RA Boriolli, JE Ferreira, RM Catarino, RYA Camargo, G Medeiros-Neto. 2004. Avaliação ultra-sonográfica da tireóide e determinação da iodúria em escolares de diferentes regiões do Estado de São Paulo. Arq Bras Endocrinol Metabol 48, 842-848.

Eiler H. 2006. Glândulas Endócrinas. In: Reece WO (ed). Dukes. Fisiologia dos Animais Domésticos. 12. Guanabara Koogan, Rio de Janeiro, Brasil, Pp 577-622. 
Khan MA, HJ Lee, WS Lee, HS Kim, SB Kim, KS Ki, JK Ha, HG Lee, YJ Choi. 2007. Pre- and postweaning performance of holstein female calves fed milk through step-down and conventional methods. J Dairy Sci 90, 876-885.

McCoy MA, JA Smyth, WA Ellis, JR Arthur, DG Kennedy. 1997. Experimental reproduction of iodine deficiency in cattle. Vet Rec 141, 544-547.

Nakatake N, S Fukata, J Tajiri. 2011. Prediction of posttreatment hypothyroidism using changes in thyroid volume after radioactive iodine therapy in adolescent patients with Graves' disease. Int J Pediatr Endocrinol 14, 1-6.

NRC, National Resarch Council. 2001. Nutrient requirements of dairy cattle. National Academy Press, Washington, D.C., USA.

Puls R. 1994. Iodine. In: Puls R (ed). Mineral levels in animal health: diagnostic data. Sherpa International, Clearbrook, British Columbia, Canada, Pp 96-97.

Seirferle E. 1975. Nervensystem - Sinnesorgane Endokrine
Drüsen. In: Nickel R, Schummer A, Seirferle E (eds). Lehrbuch der Anatomie der Haustiere. Paul Parey, Berlin, Deutschland, Pp 322-326.

Statistix. 2003. Statistix 8.0: User's manual. Tallahasse, FL, USA.

Taeymans O, S Daminet, L Duchateau, JH Saunders. 2007. Preand post-treatment ultrasonography in hypothyroid dogs. Vet Radiol Ultrasound 48, 262-269.

Tiirats T. 1997. Thyroxine, triiodothyronine and reverse-triiodothyronine concentrations in blood plasma in relation to lactational stage, milk yield, energy and dietary protein intake in Estonian dairy cows. Acta Vet Scand 38, 339-348.

Underwood EJ, NF Suttle. 1999. Iodine. In: Underwood EJ , Suttle NF (eds). The mineral nutrition of livestock. $3^{\text {rd }}$ ed. CAB International, Wallingford, UK, Pp 343-373.

Wiesner ER, JS Matoon, TG Nyland, TW Baker. 1991. Normal ultrasonographic anatomy of the canine neck. Vet Radiol 32, 185-190. 
